\title{
Optimal selection of molecular descriptors for antimicrobial peptides classification: an evolutionary feature weighting approach
}

\author{
Jesus A. Beltran, Longendri Aguilera-Mendoza and Carlos A. Brizuela* \\ From IEEE International Conference on Bioinformatics and Biomedicine 2017 \\ Kansas City, MO, USA. 13-16 November 2017
}

\begin{abstract}
Background: Antimicrobial peptides are a promising alternative for combating pathogens resistant to conventional antibiotics. Computer-assisted peptide discovery strategies are necessary to automatically assess a significant amount of data by generating models that efficiently classify what an antimicrobial peptide is, before its evaluation in the wet lab. Model's performance depends on the selection of molecular descriptors for which an efficient and effective approach has recently been proposed. Unfortunately, how to adapt this method to the selection of molecular descriptors for the classification of antimicrobial peptides and the performance it can achieve, have only preliminary been explored.
\end{abstract}

Results: We propose an adaptation of this successful feature selection approach for the weighting of molecular descriptors and assess its performance. The evaluation is conducted on six high-quality benchmark datasets that have previously been used for the empirical evaluation of state-of-art antimicrobial prediction tools in an unbiased manner. The results indicate that our approach substantially reduces the number of required molecular descriptors, improving, at the same time, the performance of classification with respect to using all molecular descriptors. Our models also outperform state-of-art prediction tools for the classification of antimicrobial and antibacterial peptides.

Conclusions: The proposed methodology is an efficient approach for the development of models to classify antimicrobial peptides. Particularly in the generation of models for discrimination against a specific antimicrobial activity, such as antibacterial. One of our future directions is aimed at using the obtained classifier to search for antimicrobial peptides in various transcriptomes.

Keywords: Antimicrobial peptides, Feature weighting, Molecular descriptors, Classification, Multi-objective evolutionary algorithm, Peptide representation

\section{Background}

Antimicrobial peptides (AMPs) are components of the host defense mechanism against bacteria and fungi, including multi-drug resistant pathogens such as Methicillin-resistant Staphylococcus aureus and vancomycin-resistant Enterococci [1]. AMPs also exhibit other biological properties like antitumor, antiviral, and

\section{*Correspondence: cbrizuel@cicese.mx}

Computer Sciences Department, Center for Scientific Research and Higher Education of Ensenada (CICESE), Carretera Ensenada-Tijuana No. 3918, Zona Playitas, 22860 Ensenada, Mexico antiparasitic activities. With the rapid increase in number of antibiotic-resistant bacteria, AMPs have received much attention as a template for the development of new drugs for the treatment of infectious diseases.

From the computational point of view, Virtual Screening (VS) [2-4] is usually applied at early stages of the drug discovery process. It contributes to the identification of putative AMPs from large peptide libraries [3, 5]. In this context, Quantitative Structure-Activity Relationship (QSAR) is of great importance for models' generation 
to classify active (AMPs) and inactive (non-AMPs) peptides [6]. QSAR modeling defines mathematical relationship between the peptides' physicochemical properties (molecular descriptors) to their biological activity [6] to classify the activity of new peptides. Machine learning approaches are tools for the generation of models that describe this relationship from a set of peptides with known activities. Admittedly, the model's performance depends on the selection of molecular descriptors since they define the chemical space in which each peptide is projected. The selection of appropriate molecular descriptors to discriminate between AMPs and non-AMPs is a hard goal to achieve due to the large number of molecular descriptors that can be calculated in peptides and to their complex interrelationships. Furthermore, new features can be added to this large set of molecular descriptors through feature construction methods [7]. Recently, an evolutionary approach [8] was proposed for AMP recognition which combines sequence-base features such as motif and positional sequence into more complex features leading to promising results. These results motivate the inclusion of these new features to the existing set to participate in the feature selection process afterwards.

In earlier studies, the selection of molecular descriptors has often been made based on chemical intuition or observed properties that give rise to the antimicrobial activity [3, 9]. In contrast, recent works employ hand-picked features (molecular descriptors) procedures or filtering methods that independently evaluate the features according to a given criterion to select the top $k$ of them [8-11]. However, these approaches present some disadvantages considering that the biological activity of peptides depends on complex interrelationships of many molecular descriptors. Therefore, we need a more rigorous feature selection procedure to improve the performance of AMPs classification [12].

Feature selection methods can be categorized into three major classes based on the features' assessment: filter, wrapper, and hybrid. First, in the filter methods, the quality of features is evaluated from the data, ignoring the effect of the selected features on the classifier algorithm performance [13]. Examples of evaluation functions used on filter methods are distance, information, and dependence measure [14]. Second, the wrapper methods incorporate the classifier's performance (e.g., error rate, accuracy) to evaluate the quality of the selected features [13]. Finally, hybrid methods combine both, the filter and wrapper methods [15]. Wrappers usually outperform filter methods, mainly because the selection of optimal features is biased towards the effect of these features on the classifier's performance. Additionally, wrapper methods have a high computational cost because they require to induce and test a classifier for each evaluated features' subset. In contrast, since filter methods are independent of classification algorithms, they may be computed efficiently [13]. Furthermore, filter methods can improve their performances by using evaluation measures for a specific classification algorithm [13]. For example, the intra-class distance could be appropriate for the instancebased learning algorithms, whereas the information gain for the decision trees classifiers.

An efficient and effective filter approach for the selection of features, based on their weighting has been recently proposed [16]. In this approach, the weights are assigned in such a manner that objects in different classes tend to be far away from each other, whereas objects within the same class tend to be close together. Unfortunately, there is a trade-off between these distances and, that is why the feature weighting challenge is modeled as a multi-objective optimization problem. In a recent work [17] we applied this formulation to the antimicrobial peptides classification problem and improved it by taking into account that molecules with similar structure tend to possess similar biological activity [18]. The central idea is that it makes no sense to minimize the distance among non-AMPs as it should be done [16], since they may have different biological activities. The proof-of-concept of our formulation in [17] showed a good performance capability for the binary classification of AMPs. The present work builds upon our improved formulation [17] and extends its results. Besides dealing with a significantly larger dataset, the statistical significance of the observed difference is assessed. We now also show the ability of our proposal to classify a subset of AMPs that explicitly targeted bacteria.

\section{Problem statement}

The general problem to solve is referred to as feature weighting problem [19], and it is known to be NP-Hard [20]. For our purposes, we model this problem as a multiobjective optimization problem (MOP) to find a set of weight vectors that simultaneously minimize the distance between AMPs and maximize the distances between AMPs and non-AMPs. To define the MOP, we follow a similar approach to the one presented in [16], where the main differences are as follows: first, the general problem of weighting feature in [16] simultaneously minimizes the intra-class distance for all classes. Instead, our approach [17] minimizes only the intra-class distance of AMPs, since the non-AMPs set might contain peptides with different biological activities, thus trying to reduce the intraclass distance for non-AMP would be contradictory with the similarity property principle [18]. Furthermore, in our approach, the number of non-zero weights are used as a tiebreaker criterion for the weight vectors with the same intra or inter-class distances. 


\section{Notation and definitions}

Before presenting the formal definition of the problem, some notation, and definitions are introduced.

- $\mathcal{X}$ is a feature set $\left\{X_{1}, \ldots, X_{m}\right\}$. In this paper, we use, without distinction, the term molecular descriptor and feature.

- $\mathcal{Y}$ is the set of class labels $\left\{C_{1}, \ldots, C_{c}\right\}$, with $c$ the number of classes. For instance, $\mathcal{Y}=\left\{C_{1}, C_{2}\right\}$, with $C_{1}=$ "AMP" and $C_{2}=$ "Non-AMP".

- $\mathcal{D}$ is the training dataset composed of $n$ peptides with a known biological activity $\left\{\left(\mathbf{x}_{1}, y_{1}\right), \ldots,\left(\mathbf{x}_{n}, y_{n}\right)\right\}$, where $\mathbf{x}_{i}$ is an $m$-dimensional vector $\left[x_{i 1}, \ldots, x_{i m}\right]^{T}$ that captures the physicochemical properties into real values, each component $x_{i j}$ encodes the value for the $j$ th molecular descriptor (i.e., feature) of the ith peptide sequence. $y_{i} \in \mathcal{Y}$ denotes whether $\mathbf{x}_{i}$ has the antimicrobial activity or not. $\mathcal{D}$ can be expressed as a matrix with $n \times(m+1)$ elements whose rows are given by $\mathbf{x}_{i}^{T}$ and $y_{i}$.

$$
\mathcal{D}=\left[\begin{array}{cccc|c}
x_{11} & x_{12} & \cdots & x_{1 m} & y_{1} \\
x_{21} & x_{22} & \cdots & x_{2 m} & y_{2} \\
\vdots & \ddots & \vdots & \vdots \\
x_{n 1} & x_{n 2} & \cdots & x_{n m} & y_{n}
\end{array}\right]
$$

This data matrix $\mathcal{D}$ is also known as a descriptor matrix [21].

- $\mathbf{w}=\left[w_{1}, \ldots, w_{m}\right]^{T}$ is a weight vector that specifies the rescaling value of each feature, the corresponding weight for the ith feature is given by [16]:

$$
w_{i}= \begin{cases}{[1, \mathcal{A}]} & \text { if the feature } X_{i} \text { is selected; } \\ 0 & \text { if the feature } X_{i} \text { is rejected }\end{cases}
$$

where $\mathcal{A}$ is the maximum weight for $w_{i}$ and it takes any positive real number. As in [16], $\mathcal{A}=10$ in this work.

- The weighted distance (also known as weighted Manhattan distance) between two data points $\mathbf{x}_{p}$ and $\mathbf{x}_{q}$ is defined as:

$$
d\left(\mathbf{w}, \mathbf{x}_{p}, \mathbf{x}_{q}\right)=\sum_{i=1}^{m} w_{i}\left|x_{p i}-x_{q i}\right|
$$

where |.| represents the $L_{1}$ norm. Let $y=A M P$ the class label of interest, then the intra-class distance for the class of interest is defined as follows:

$$
D_{\text {intra }}(\mathbf{w}, \mathcal{D})=\sum_{p=1}^{n-1} \sum_{\substack{q=p+1 \\ y_{p}, y_{q}=A M P}}^{n} d\left(\mathbf{w}, \mathbf{x}_{p}, \mathbf{x}_{q}\right)
$$

Additionally, the inter-class distance is defined as:

$$
D_{\text {inter }}(\mathbf{w}, \mathcal{D})=\sum_{p=1}^{n-1} \sum_{\substack{q=p+1 \\ y_{p} \neq y_{q}}}^{n} d\left(\mathbf{w}, \mathbf{x}_{p}, \mathbf{x}_{q}\right)
$$

A multi-objective approach to the feature weighting problem Let $\mathcal{D}$ be a training dataset with $n$ instances and $m$ candidate input features, we assume that for each instance $\mathbf{x}_{i}^{T} \in \mathcal{D}$, the value $x_{i j}$ is in the interval $[1, \mathcal{A}]$, where $x_{i j}$ is the $j$-th component of the vector $\mathbf{x}_{i}^{T}$. Then, the multiobjective feature weighting problem can be stated as:

$$
\begin{array}{ll}
\underset{\mathbf{w}}{\operatorname{minimize}} & F(\mathbf{w})=\left[f_{1}(\mathbf{w}), f_{2}(\mathbf{w})\right]^{T} \\
\text { subject to } & w_{i} \in\{0\} \cup[1, \mathcal{A}] i=1, \ldots, m,
\end{array}
$$

where,

$$
\begin{aligned}
& f_{1}(\mathbf{w})=D_{\text {intra }}(\mathbf{w}, \mathcal{D})+\frac{[\min \{1, \mathbf{w}\}]^{T} \mathbf{1}}{m}, \\
& f_{2}(\mathbf{w})=-D_{\text {inter }}(\mathbf{w}, \mathcal{D})+\frac{[\min \{1, \mathbf{w}\}]^{T} \mathbf{1}}{m},
\end{aligned}
$$

here, the term $[\min \{1, \mathbf{w}\}]^{T} \mathbf{1}$ is the number of weights that are different from zero (i.e., $w_{i}>0$ for $i=1, \ldots, m$.). This term promotes a weight vector with a smaller number of features than any other weight vector with the same intraclass or inter-class distances.

\section{Results}

To evaluate the effectiveness of our approach, called Multi-Objective Approach for Feature Weighting (MOEA-FW), we conducted experiments on six highquality benchmark datasets that have recently been used for empirical evaluation of state-of-art antimicrobial prediction tools in an unbiased manner [12]. These datasets were selected because they are composed of manually curated and experimentally validated AMPs; in these datasets, the non-AMPs have the same peptide distribution as that observed in AMPs (see "Methods" section). This experimental study was divided into four parts. In the first part, we aimed at selecting the appropriate molecular descriptors for each dataset through their scaling. Whereas, in the second part, different classification models are induced by four machine learning algorithms (MLAs) with the transformed datasets. In the third part, the best classification models generated were used to predict the antimicrobial activity for new peptides sequences, i.e., peptide sequences that have not been used either for obtaining the weight vectors or for the cross-validation test to choose the best classifiers. Finally, we compared our result with those presented in a recent work [12] that evaluates different AMP predictors.

\section{Performance measure}

To compare the best compromise solutions found by our MOEA-FW algorithm, for each dataset, a performance estimation method was employed to evaluate the efficiency of the model to classify antimicrobial peptides. The performance estimation method employed 10-fold 
Cross-Validation (10-fold CV) as a re-sampling method and a diverse set of evaluation metrics. In the 10-fold CV, the dataset is partitioned into 10 non-empty disjoint subsets (i.e., fold); each subset has roughly equal size. Nine folds are employed for the machine learning algorithm to induce a classifier, and the classifier is tested on the remaining subset, this procedure is repeated ten times. Additionally, the performance of the classifier was estimated by using the average values from the tests. To test the classification performance, the following metrics were used: accuracy, Matthews correlation coefficient, precision, specificity, sensitivity, balance accuracy, and the area under a ROC Curve (AUC). We consider the instances of the class AMP as positive and the instances of the class non-AMP as negative; then the metrics can be formally defined as follows:

- Accuracy (Acc) [22]:

$$
A c c=\frac{T P+T N}{T P+T N+F P+F N}
$$

- Matthews correlation coefficient (MCC) [22]:

$$
M C C=\frac{T P \times T N-F N \times F P}{\sqrt{(T P+F N)(T N+F P)(T P+F P)(T N+F N)}}
$$

- Precision (Prec):

$$
\text { Prec }=\frac{T P}{T P+F P}
$$

- Sensitivity (Sens):

$$
\text { Sens }=\frac{T P}{T P+F N}
$$

- Balance Accuracy (Bal Acc) [12]:

$$
\text { BalAcc }=\frac{1}{2}\left(\frac{T P}{T P+F N}\right)+\frac{1}{2}\left(\frac{T N}{T N+F P}\right)
$$

where TP, TN, FP, and FN are the number of true positive, true negative, false positive, and false negative, respectively. Given that the considered datasets are imbalanced classes (i.e., the AMPs and non-AMPs are not represented equally into the datasets), we used the balance accuracy and the AUC to obtain a better measure of the induced-models' performance.

\section{Weighting of molecular descriptors}

Figure 1 displays the consolidated non-dominated front obtained by our approach (MOEA-FW) for each dataset. The consolidated non-dominated front is generated after 30 independent runs of MOEA-FW. The diamond and square marker (i.e., $\lambda_{1}=0.55$ and $\lambda_{1}=0.6$ ) represent the values for the best compromise solutions that encourage the objective $f_{1}$ (i.e., minimize the distance between peptides with antimicrobial activity). Alternatively, $\lambda_{1}=0.45$ and $\lambda_{1}=0.4$ represent the values for the best compromise solutions that encourage the objective $f_{2}$ (i.e., maximize the distance between AMPs and non-AMPs). Furthermore, $\lambda_{1}=0.5$ represents the value for the best compromise solution where both objectives are equally important.

The percentage of number of molecular descriptors reduction shown in Fig. 2 indicates a similar behavior on the six datasets for each best compromise solution. In particular, the best compromise solution $\lambda_{1}=0.5$ has, on average, a reduction in the number of molecular descriptors of $52.7 \%$, i.e., on average, the best-weighted solution has 128 features out of 272 . Nevertheless, the DAMP_BACTERIOCIN dataset shows an increment of this measure for solution $\lambda_{1}=0.45$. These findings indicate that solutions supporting the objective $f_{1}$ (i.e., inter-class distance) have, on average, fewer molecular descriptors than those that support the objective $f_{2}$ (i.e., intra-class distance).

\section{Model selection}

Next, for each best compromise solution earlier obtained (i.e., a weight vector $\mathbf{w}$ ), the original datasets were transformed (i.e., weighted; see "Methods" section). Then, for each transformed data, four classification models were constructed by the following machine learning algorithms: random forest (RF), k-nearest neighbor (KNN), multi-layer perceptron (MLP), and a linear support vector machine (SVM-L).

As mentioned earlier, the balance accuracy (BalAcc) was considered as a measure to determine the best model on the six datasets weighted by the best compromise solutions. We applied the non-parametric Friedman's test [23] and Nemenyi post hoc test [24] to verify whether there are significant differences among the classifiers' performance. The Friedman [23] and Nemenyi tests have been widely used in the literature for statistical comparison of classifiers on multiple datasets (the interested reader is referred to [24] for more information about how to perform both tests).

Our results indicated that the best compromise solution, with $\lambda_{1}=0.5$, allows to induce on average, better classification models regardless the machine learning algorithm, the BalAcc was $87.52 \%$ (see Additional file 1).

The statistical analysis of the MLAs' performance identified (by the Friedman test) a significant difference in the $\operatorname{BalAcc}\left(\chi_{f}^{2}(3)=55.2, p\right.$-value $\left.=6.224 \mathrm{e}-12\right)$ of the four MLAs on multiple datasets. Our results show that, on average, SVM-L ranked first (with rank 1.23), KNN second (with rank 2.43), RF third (2.63), and MLP fourth (3.7) (see Additional file 1: Table S1). Furthermore, we found that SVM-L performed significantly better than MLP (Nemenyi: $z=7.4, p$-value $=8.40 \mathrm{e}-13), \mathrm{RF}$ (Nemenyi: $z=4.2, p$-value $=0.00016$ ), and $\mathrm{KNN}$ (Nemenyi: $z=3.6$, 

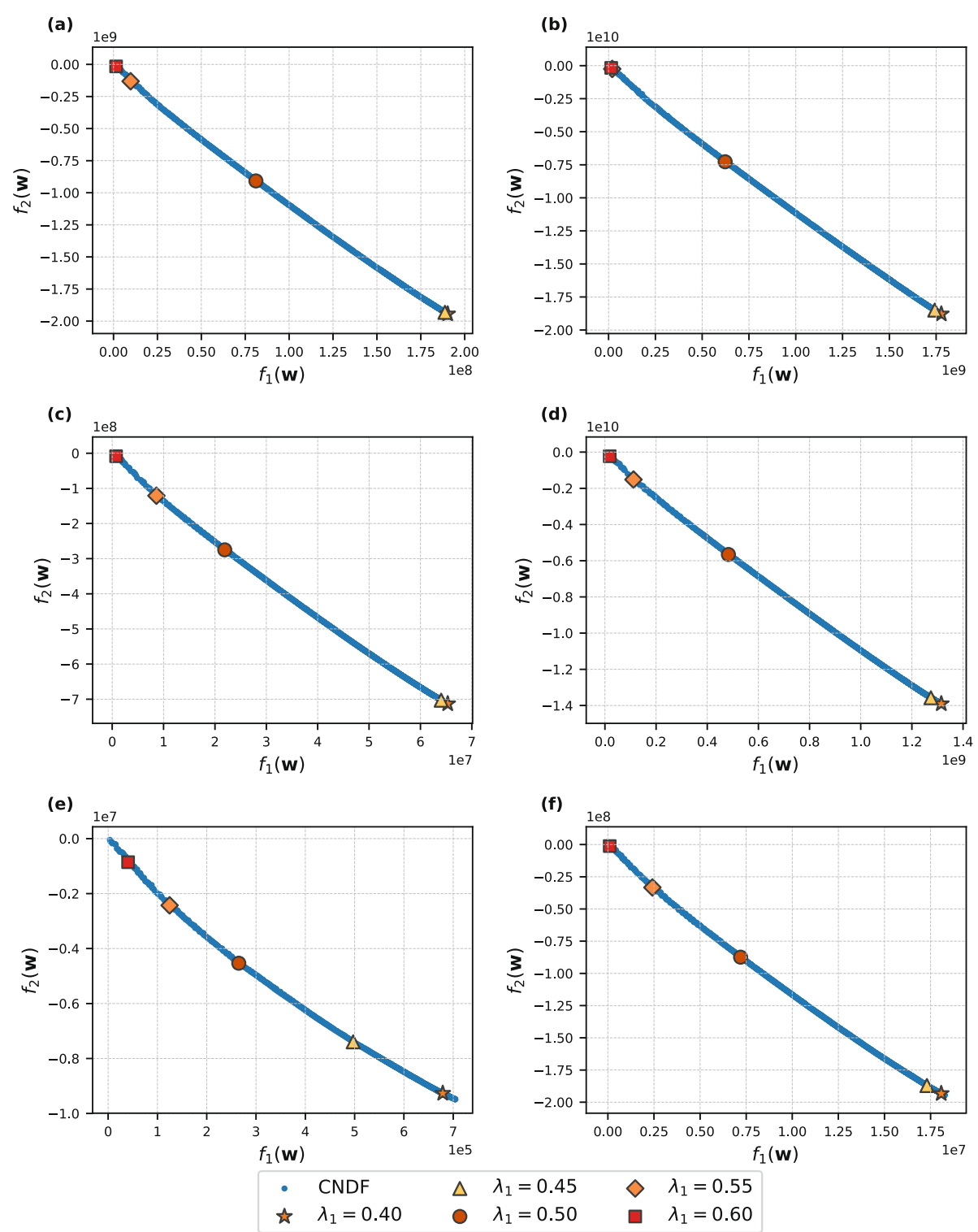

Fig. 1 The consolidated non-dominated front (CNDF) visualization. The CNDF is generated after 30 runs of the MOEA-FW approach for each dataset. The markers represent the values for the best compromise solution given $\lambda_{1}$. a DAMPD_AMP. b APD3_AMP. c DAMPD_ANTIBACTERIAL. d APD3_ANTIBACTERIAL. e DAMPD_BACTERIOCIN. f APD3_BACTERIOCIN

$p$-value $=0.00181)$. Similarly, KNN performed significantly better than MLP $(z=3.8, p$-value $=0.00083)$. Although the KNN performs a little better than RF, there was no statistical significant difference $(p$-value $=0.932)$ between them.

In particular, considering only the best compromise solution with $\lambda_{1}=0.5$, the average of BalAcc for SVML was $92.65 \%$ and for KNN 90.13\% (Summary statistics on BalAcc(\%) for all best compromise solutions can be found in the Additional file 1). Hence, our findings indicate that for the six datasets, the best compromise solution with $\lambda_{1}=0.5$ using SVM-L and KNN induced better classification models.

Table 1 summarizes the result obtained by SVM-L and $\mathrm{KNN}$ with the best compromise solution at $\lambda_{1}=0.5$ (detailed results are presented in Additional files 2, 3, 4, 5, 6 and 7). The metric's values represent the average for the 10 -fold cross-validation. In this table, a Wilcoxon test is also performed on the observed differences between KNN and SVM-L for Sens(\%), Spec(\%), Prec(\%), BalAcc, Acc(\%), MCC, and AUC values; if the difference is statistically significant, at a confidence level of $95 \%$, then an 


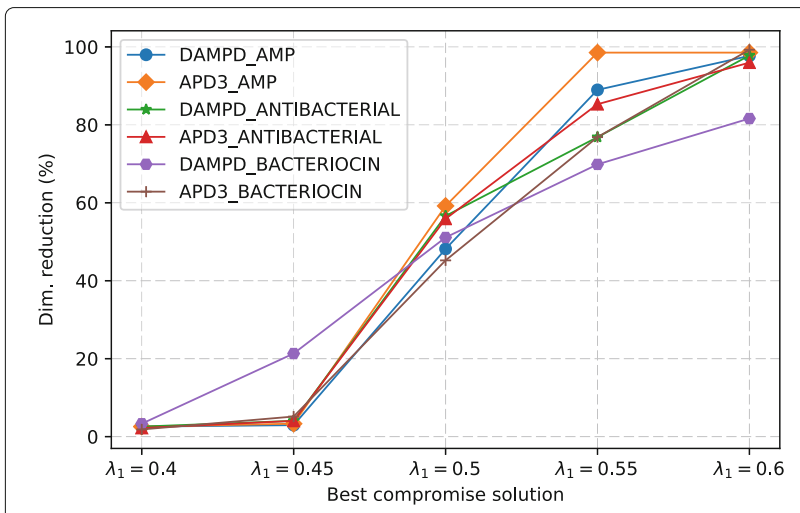

Fig. 2 Percentage of number of molecular descriptors reduction for the best compromise solutions on six datasets

asterisk is added to the winner value (in bold). In most cases, the classification models generated by the KNN showed better specificity and precision than the ones generated by the SVM-L, i.e., models correctly predict $96 \%$ of non-AMPs, and correctly classify $80 \%$ of predicted AMPs. In comparison, the classification model obtained by SVML showed good sensitivity, namely, the model correctly classifies $88.33 \%$ of AMPs.

To determine the effect of MOEA-FW on the efficiency of the model to classify AMPs for each dataset, we compared the performance of two classifiers generated by the same machine learning algorithm, one applying the MOEA-FW and the other one, by using all candidate input features (i.e., baseline). We selected the best machine learning algorithm per database, this is according to the balanced accuracy column in Table 1 . We run the Wilcoxon's test on the BalAcc resulting from the 10-fold cross-validation of our proposed method and the baseline for each dataset (Additional files 2, 3, 4, 5, 6 and 7). The models generated by MOEA-FW shows a significant improvement over the baseline models on the BalAcc. For each dataset, the significant difference in BalAcc between MOEA-FW and baseline were as follows: DAMPD_AMP $(p$-value $=0.00976)$, APD3_AMP $(p$-value $=0.00195)$, DAMPD_ANTIBACTERIAL $(p$-value $=0.00195)$, APD3_ANTIBACTERIAL $(p$-value $=0.00976)$, DAMPD_BACTERIOCIN $(p$-value $=0.051)$, and APD3_BACTERIOCIN ( $p$-value $=0.08398)$. Similar results were observed for the other metrics, they are summarized in Fig. 3. In this figure, an asterisk indicates that the observed different is statistically significant.

On the other hand, if we take into consideration other metrics (i.e., Sens, Spec, AUC, MCC, Pres, Acc) to compare both models, the results show that the models generated by using MOEA-FW achieve a comparable or superior performance than those obtained by using all candidate input features. In particular, for the datasets DAMPD_AMP, APD3_AMP, and APD3_ANTIBACTERIAL, the MOEA-FW shows an improvement over the baseline (see Fig. 3). In contrast, datasets DAMPD_BACTERIOCIN and APD3_BACTERIOCIN showed a decrease in the precision measure with respect to the baseline. This result suggests that our proposal cannot find a suitable chemical space for BACTERIOCIN datasets, whereby an efficient

Table 1 10-Fold Cross-Validation performance on six datasets for KNN and SVM-L, $\lambda_{1}=0.5$

\begin{tabular}{|c|c|c|c|c|c|c|c|c|}
\hline Dataset & MLA & Sens(\%) & Spec(\%) & Prec(\%) & Bal Acc(\%) & $A c c(\%)$ & MCC & AUC \\
\hline \multirow[t]{2}{*}{ DAMPD_AMP } & KNN & 71.97 & 97.22* & $83.75^{*}$ & 84.60 & 93.01 & 0.735 & 0.846 \\
\hline & SVM-L & $\mathbf{8 8 . 0 7}^{* a}$ & 92.30 & 69.56 & 90.19* & 91.62 & 0.734 & $0.902 *$ \\
\hline \multirow[t]{2}{*}{ APD3_AMP } & KNN & 80.85 & $95.27^{*}$ & 77.23* & 88.06 & 92.85 & 0.747 & 0.881 \\
\hline & SVM-L & $91.65^{*}$ & 92.53 & 70.75 & 92.09* & 92.36 & 0.762 & $0.921 *$ \\
\hline \multirow[t]{2}{*}{ DAMPD_ANTIBACTERIAL } & KNN & 91.04 & 96.45 & 84.37 & 93.75 & 95.51 & 0.849 & 0.937 \\
\hline & SVM-L & 88.49 & 96.54 & 84.18 & 92.51 & 95.06 & 0.832 & 0.925 \\
\hline \multirow[t]{2}{*}{ APD3_ANTIBACTERIAL } & KNN & 79.32 & 95.30* & 77.18* & 87.31 & 92.61 & 0.738 & 0.873 \\
\hline & SVM-L & 91.34* & 92.22 & 70.33 & 91.78* & 92.07 & 0.756 & $0.918^{*}$ \\
\hline \multirow[t]{2}{*}{ DAMPD_BACTEROCIN } & KNN & 100 & 95.53 & 85.83 & 97.76 & 96.36 & 0.902 & 0.978 \\
\hline & SVM-L & 100 & 98.89 & 96.67 & 99.44 & 99.09 & 0.977 & 0.994 \\
\hline \multirow[t]{2}{*}{ APD3_BACTEROCIN } & KNN & 83.50 & 95.04 & 77.05 & 89.27 & 93.12 & 0.758 & 0.893 \\
\hline & SVM-L & 85.38 & 94.83 & 77.28 & 90.10 & 93.12 & 0.768 & 0.901 \\
\hline
\end{tabular}

Each value is the average performance from 10-fold cross-validation by the classifier built by the machine learning algorithm (second column) on the dataset (first column). Wilcoxon signed rank test was performed on the measure resulting from the 10 -fold cross-validation of KNN and SVM-L. The models with significant improvement at $p$-value $\leq 0.05$ are marked with the symbol *

${ }^{a}$ Bold font indicates the best value per measure for every dataset 

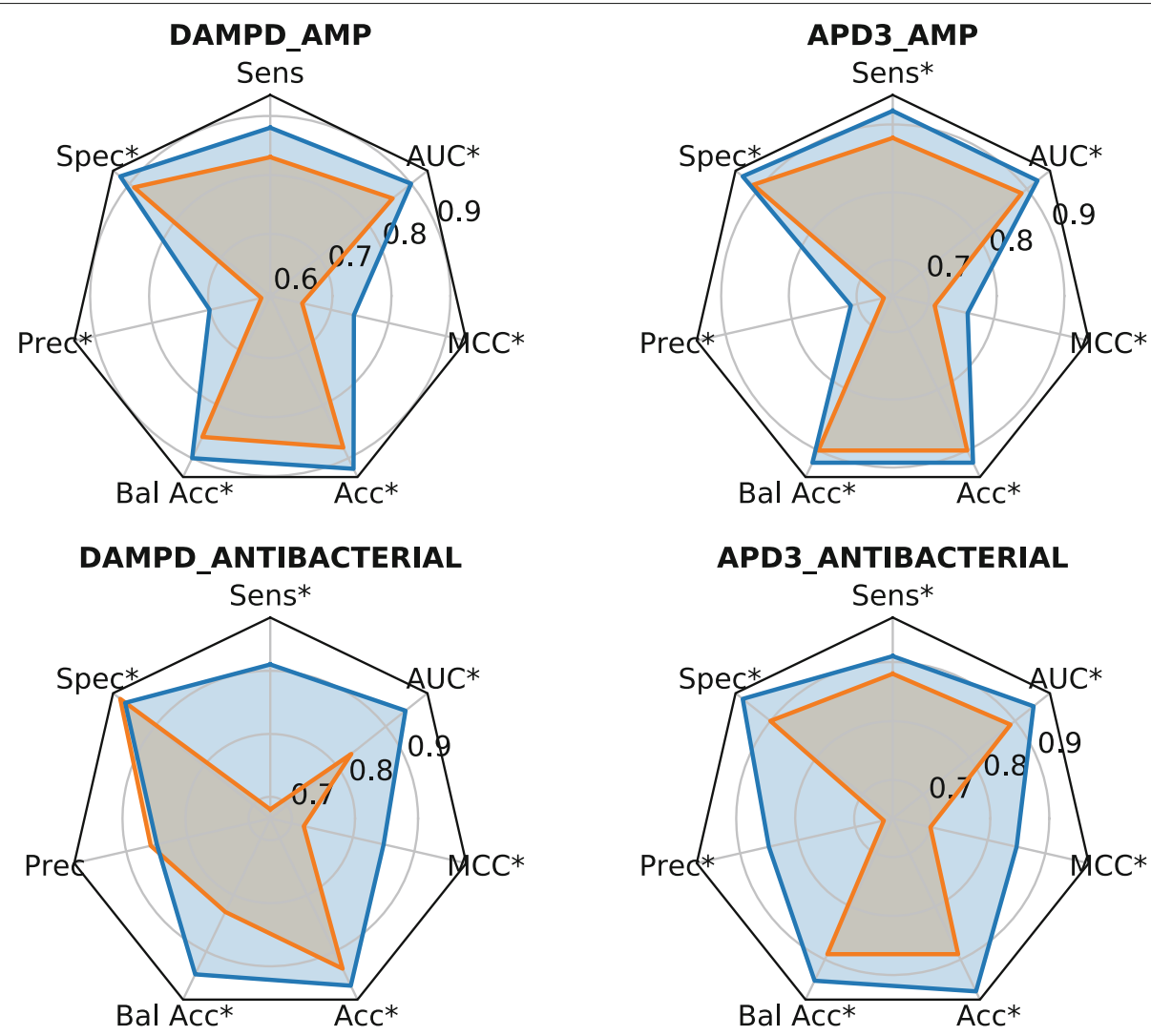

DAMPD_BACTERIOCIN
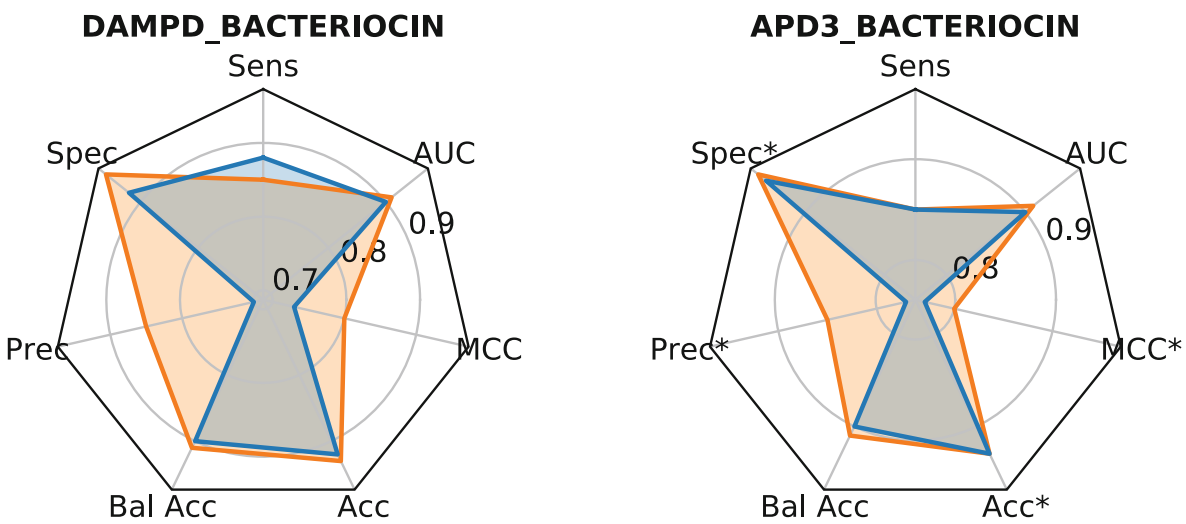

Baseline

Proposed $\left(\lambda_{1}=0.5\right)$

Fig. 3 Performance comparison between the best model achieved by MOEA-FW and the baseline. Each plot shows the performance measure by 10-fol cross-validation of the best model achieved by MOEA-FW and the baseline (i.e., all candidate input features) for a particular dataset. The polygon represents a particular performance's model. When a polygon is covered means that the model is worse in all metrics that the model represented by the polygon that includes it. Wilcoxon signed rank test was performed on the measure resulting from the 10-fold cross-validation of best model achieved by MOEA-FW and the baseline. The models with significant improvement at $p$-value $\leq 0.05$ are marked with the symbol *

model could be induced to discriminate what a bacteriocin is. Conjectures of why this is happening are given in the "Discussion" section.

\section{Model assessment}

After selecting the best models obtained with the best compromise solution given $\lambda_{1}=0.5$, and using KNN 
and SVM-L, we measured their prediction capacity over new peptide sequences, this is, peptide sequences that have not been used either for obtaining the weight vectors or for cross-validation tests to choose the best classifiers (see "Methods" section). We observed that all classifiers induced by SVM-L have an AUC value $>0.83$, this means that the models generated by SVM-L have an excellent capacity to learn what an antimicrobial peptide is. Whereas, the model generated by KNN maintain an excellent specificity (as the results presented in Table 1 indicate).

On the other hand, comparing the results for DAMP_BACTIBASE set, espacially for bacteriocin, in Tables 1 and 2, the considerable difference in sensitivity (Sens(\%)) may be because of the small number of bacteriocins in the test set.

\section{Comparison with existing AMP classifiers}

The best model generated by our approach MOEA-FW was compared with others AMP predictors that used the same datasets. It is important to note that the number of instances between our test and the test showed in [12] are different, because in [12] the evaluation of AMP's predictors was performed by using the full examples of the six datasets, whereas in our method, we used only $20 \%$ of them (i.e., the other $80 \%$ of the dataset was used in the optimization process, see "Methods" section). However, this comparison is intended to observe the predictive capacity of the classification models generated with our approach and those presented by the state-of-the-art methods.
The classifier performances presented in this work and those reported by state-of-art methods for the AMP prediction are summarized in Tables 3 and 4. Our results reflect that the models produced by our approach have a better performance than the state-of-the-art methods for the classification of antimicrobial and antibacterial peptides. It is worth noting that models derived from our approach to classify antibacterial peptides outperformed AntiBP [25] and AntiBP2 [26] (see Tables 3 and 4). However, our method is improved by BAGEL3 [27] for the BACTERIOCIN datasets.

\section{Discussion}

Our approach aims to identify a weight for each molecular descriptor, in such manner that, peptides with antimicrobial activity tend to be close together, whereas peptides with different biological activities tend to be far away from each other. Our results indicate that the best compromise solution with $\lambda_{1}=0.5$ allows, on average, the best balance accuracy for all six databases. Furthermore, this solution allows a reduction of at least $52 \%$ in the number of molecular descriptors. It is important to note that in our previous work [17], the best solution, for a smaller database, was found with $\lambda_{1}=0.55$, and it reduced the number of descriptors by $67.90 \%$. The difference may be a consequence of having unbalanced datasets in this case. With the best compromise solution $\left(\lambda_{1}=0.5\right)$, we transform (weight the features) the datasets and build models for the binary classification of AMPs and non-AMPs. Our results indicate that both KNN and SVM-L allow to achieve reliable models for classification of antimicrobial

Table 2 Performance comparison of KNN and SVM-L on unseen sequences from the six datasets, $\lambda_{1}=0.5$

\begin{tabular}{|c|c|c|c|c|c|c|c|c|}
\hline Dataset & $M L$ & Sens(\%) & Spec(\%) & Prec(\%) & Bal Acc(\%) & $A c c(\%)$ & MCC & AUC \\
\hline \multirow[t]{2}{*}{ DAMPD_AMP } & KNN & 72.16 & 94.17 & 68.63 & 83.17 & 90.87 & 0.650 & 0.832 \\
\hline & SVM-L & $77.32^{\mathrm{a}}$ & 91.62 & 61.98 & 84.47 & 89.47 & 0.631 & 0.845 \\
\hline \multirow[t]{2}{*}{ APD3_AMP } & KNN & 70.82 & 92.11 & 65.10 & 81.47 & 88.45 & 0.609 & 0.815 \\
\hline & SVM-L & 89.24 & 82.87 & 51.98 & 86.05 & 83.97 & 0.597 & 0.861 \\
\hline \multirow[t]{2}{*}{ DAMPD_ANTIBACTERIAL } & KNN & 80.0 & 90.91 & 60.27 & 85.45 & 89.30 & 0.634 & 0.855 \\
\hline & SVML & 74.55 & 93.10 & 65.08 & 83.82 & 90.37 & 0.640 & 0.838 \\
\hline \multirow[t]{2}{*}{ APD3_ANTIBACTERIAL } & KNN & 65.97 & 93.91 & 68.35 & 79.94 & 89.26 & 0.607 & 0.799 \\
\hline & SVM-L & 81.94 & 91.55 & 65.92 & 86.75 & 89.95 & 0.676 & 0.867 \\
\hline \multirow[t]{2}{*}{ DAMPD_BACTEROCIN } & KNN & 80 & 87.50 & 50.00 & 83.75 & 86.49 & 0.561 & 0.838 \\
\hline & SVM-L & 60 & 96.88 & 75.00 & 78.44 & 91.89 & 0.626 & 0.784 \\
\hline \multirow[t]{2}{*}{ APD3_BACTEROCIN } & KNN & 75.86 & 94.23 & 70.97 & 85.05 & 91.35 & 0.682 & 0.850 \\
\hline & SVM-L & 93.10 & 92.95 & 71.05 & 93.03 & 92.97 & 0.774 & 0.930 \\
\hline
\end{tabular}

"Each value is the performance on the testing dataset by the classifier built by the machine learning algorithm (second column) on the dataset after applying the best compromise solution for $\lambda_{1}=0.5$ (first column)

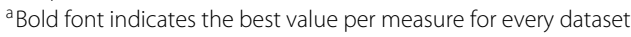


Table 3 Performance comparison among the AMPs prediction methods reported in [12] with our proposed approach for the DAMPD dataset

\begin{tabular}{|c|c|c|c|c|c|}
\hline Tool & Task & Sens(\%) & Spec(\%) & $\operatorname{Prec}(\%)$ & Bal Acc(\%) \\
\hline MOEA-FW(SVM-L) & Antimicrobial & 77.32 & 91.62 & 61.98 & 84.47 \\
\hline CAMPR3(RF) & & $92.32^{\mathrm{a}}$ & 72.65 & 40.30 & 82.49 \\
\hline CAMPR3(SVM) & & 90.13 & 72.10 & 39.25 & 81.11 \\
\hline ADAM & & 84.09 & 68.88 & 35.09 & 76.49 \\
\hline MLAMP & & 63.62 & 82.27 & 41.78 & 72.94 \\
\hline DBAASP & & 22.12 & 92.87 & 38.28 & 57.49 \\
\hline AMPA & & 48.81 & 84.79 & 39.09 & 66.80 \\
\hline MOEA-FW(KNN) & Antibacterial & 80.00 & 90.91 & 60.27 & 85.45 \\
\hline AntiBP & & 89.78 & 45.05 & 24.63 & 67.41 \\
\hline AntiBP2 & & 86.90 & 15.97 & 17.14 & 51.44 \\
\hline MOEA-FW(KNN) & Bacteriocin & 80.00 & 87.50 & 50.00 & 83.75 \\
\hline BAGEL3 & & 93.55 & 100.0 & 100.0 & 96.77 \\
\hline BACTIBASE & & 83.87 & 100.0 & 100.0 & 91.93 \\
\hline
\end{tabular}

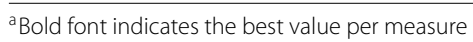

and antibacterial peptides. These results support the idea that our MOEA-FW approach allows generating better models for a specific antimicrobial activity, in this particular case, antibacterial activity. In this direction, we expect to use this approach in the future, to classify other specific antimicrobial activities, such as antiviral, anti-fungal, and anti-parasitic, accordingly to determine whether this classification performance is also observed in those particular antimicrobial activities.

As mentioned earlier, the models generated by KNN achieve high specificity and precision, while models induced by SVM-L produce high sensitivity (see Table 1).
These results suggest that, combining the models generated by KNN and SVM-L, we could exploit their properties to generate even more efficient models.

On the other hand, the lowest performance model generated by MOEA-FW was for the classification of peptides which source and target are bacteria (i.e., bacteriocins). In this case, our approach was not able to produce a chemical space where both, the peptide activity and their source could be discriminated. It is important to note, that BAGEL3 [27] and BACTIBASE [28] use properties related to sequence similarity to classify bacteriocins.

Table 4 Performance comparison among the AMPs prediction methods reported in [12] with our proposed approach for the APD3 dataset

\begin{tabular}{|c|c|c|c|c|c|}
\hline Tool & Task & Sens(\%) & Spec(\%) & $\operatorname{Prec}(\%)$ & Bal Acc(\%) \\
\hline MOEA-FW(SVM-L) & Antimicrobial & 89.24 & 82.87 & 51.98 & 86.05 \\
\hline CAMPR3(RF) & & $94.80^{\mathrm{a}}$ & 72.65 & 40.30 & 82.49 \\
\hline CAMPR3(SVM) & & 90.60 & 72.10 & 39.25 & 81.11 \\
\hline ADAM & & 91.07 & 68.88 & 35.09 & 76.49 \\
\hline MLAMP & & 75.59 & 82.27 & 41.78 & 72.94 \\
\hline DBAASP & & 62.81 & 92.87 & 38.28 & 57.49 \\
\hline AMPA & & 39.17 & 84.79 & 39.09 & 66.80 \\
\hline MOEA-FW(SVM-L) & Antibacterial & 81.94 & 91.55 & 65.92 & 86.75 \\
\hline AntiBP2 & & 66.59 & 26.00 & 15.25 & 46.30 \\
\hline MOEA-FW(SVM-L) & Bacteriocin & 93.10 & 92.95 & 71.05 & 93.03 \\
\hline BAGEL3 & & 86.36 & 100.0 & 100.0 & 93.18 \\
\hline BACTIBASE & & 38.36 & 100.0 & 100.0 & 69.48 \\
\hline
\end{tabular}

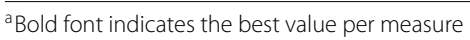




\section{Conclusions}

This work deals with the problem of molecular descriptors weighting by modeling it as a multi-objective optimization problem, such that peptides with different biological activities tend to be far away from each other, whereas, AMPs tend to be close together. To solve this problem, a variant of a general methodology [16] based on a multi-objective evolutionary algorithm (MOEA/D-DE) $[29,30]$ was employed. Also, we introduce a multi-criteria decision-making approach to select the weight vectors with different degrees of satisfaction between the intraclass and inter-class distances for the target class. Then, with these weight vectors, we scaled the datasets where the peptides are represented by molecular descriptors, and generated different models for the binary classification of AMPs. The analysis of experimental results, on six unbalanced datasets, indicates that the proposed methodology is effective on the development of models to predict antimicrobial peptides. Particularly, in the generation of models for discrimination against a specific antimicrobial activity, such as antibacterial. Given this last result, future research direction aims at constructing classifiers that specialize in specific antimicrobial activities such as antiviral, antifungal, antitumor, among others.

\section{Methods}

The scheme of the methodology adopted in this study is shown in Fig. 4. Each process is described in detail in this section, including selection and splitting of a dataset, computing and preprocessing of molecular descriptors, molecular descriptor weighting, and classification of antimicrobial peptides.

\section{Data collection}

For this study, we used six sets of peptide sequences, for which AMPs are experimentally validated whereas non-AMPs were randomly selected from a supersequence generated from the concatenation of proteins retrieved from UniProt. None of the retrieved proteins have been annotated as antimicrobial, and some of them are intracellular. From the supersequence, six non-AMPs are randomly extracted for each AMP in the dataset [12]. The datasets were obtained from the publicly available supplementary data of a recent work [12]. Then, we removed the peptide sequence that contains non-standard residues (e.g., peptide sequences with undetermined amino acids such as ' $\mathrm{X}$, ' $\mathrm{B}$ ', 'J' or ' $\mathrm{Z}$ '). We named these datasets according to i) the database from which the AMPs were recovered and, ii) their annotated activity. Regarding their database, we named the datasets DAMPD and APD3, because they come from the Dragon Antimicrobial Peptide Database (DAMPD) [31] and the Antimicrobial Peptide Database (APD3) [32], respectively. Regarding their annotated activity, we named the datasets as AMP, ANTIBACTERIAL and BACTERIOCIN. AMP are peptides that have antimicrobial activity. ANTIBACTERIAL is a proper subset of AMP since they are antimicrobial peptides that explicitly targeted bacteria. Additionally, BACTERIOCIN is a proper subset of ANTIBACTERIAL, the source organisms of such peptides are also bacteria (these peptides are referred to as bacteriocins, the interested reader is referred to [33] for more information on peptide naming and classification).

Each dataset was split into two random parts, training and test sets. The training set contains $80 \%$ of randomly selected sequences from the original dataset, while the test set contains the remaining sequences (see Table 5). The training set is used in the next steps of this section, while the test set is only used to test the effectiveness of the models generated by our approach.

\section{Computation of molecular descriptors}

Molecular descriptors are derived from a logical and mathematical procedure which transform physical and

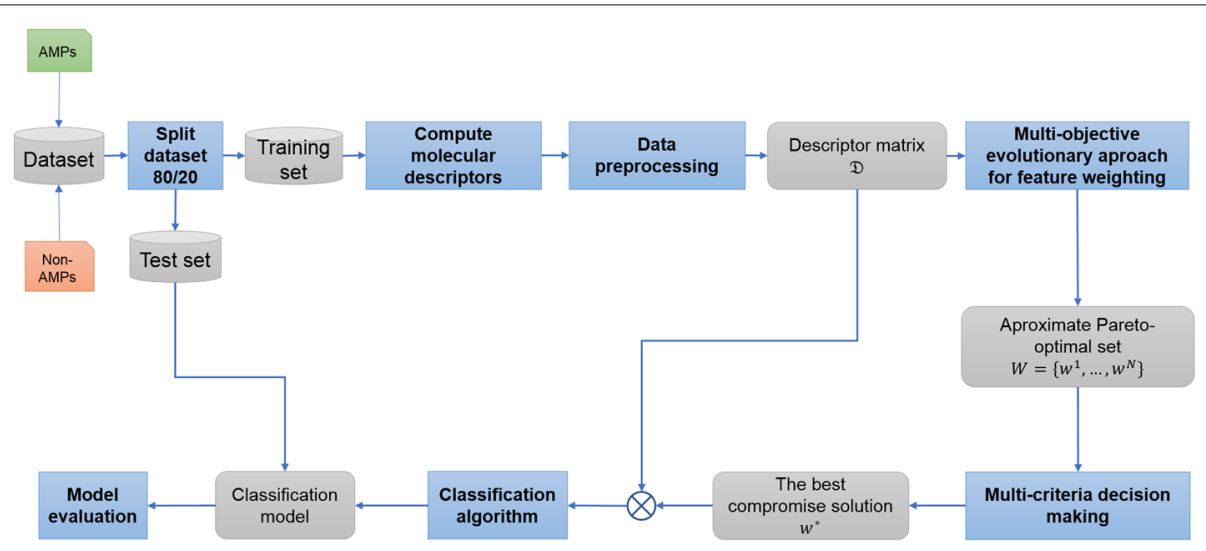

Fig. 4 The overall scheme of the feature weighting framework. The rectangles with bold texts represents processes, and the rounded rectangles represent the inputs and outputs of processes 
Table 5 Summary of peptide datasets

\begin{tabular}{llll}
\hline Dataset & $\begin{array}{l}\text { No. of AMP } \\
\text { sequences }\end{array}$ & $\begin{array}{l}\text { No. of Non-AMP } \\
\text { sequences }\end{array}$ & Total \\
\hline DAMPD_AMP & 438 & 2174 & 2612 \\
DAMPD_ANTIBACTERIAL & 255 & 1242 & 1497 \\
DAMP_BACTEROCIN & 24 & 123 & 147 \\
APD3_AMP & 1360 & 6860 & 8220 \\
ADP3_ANTIBACTERIAL & 1158 & 5777 & 6935 \\
ADP3_BACTEROCIN & 125 & 612 & 737 \\
\hline "The datasets were extracted from [12] and we removed the sequences with \\
non-standard residues
\end{tabular}

chemical information encoded in a molecule representation into useful numbers [34]. Nowadays, there are many proposed descriptors, that can be grouped according to their dimensionality from $0 D$ to $3 \mathrm{D}$. The $0 \mathrm{D}$ descriptors are very simple molecular properties (e.g., molecular mass and atom count), that depend only on the molecular composition of the peptide. The 1D descriptors encode information about molecular structural fragments (e.g., distance between two cysteine residues, hydrophobic moment). The 2D descriptors are also known as topological descriptors, and they give us information contained in a molecular graph (e.g., Weiner index). Furthermore, 3D descriptors capture the molecular geometry, stereochemical, and surface properties [6].

Two free software packages were used to extract molecular descriptors: Tango [35-37] and the in-house Java Peptide Descriptor from Sequences (JPEDES) tool [17]. The first one was used to compute the following physicochemical properties: $\alpha$-helix propensity, $\beta$-sheet propensity, turn structure propensity, and in vitro aggregation. Whereas, JPEDES [17] was used to codify OD and 1D descriptors. Unfortunately, the 3D descriptors were not computed due to unavailability 3D-structures for most known AMPs. Altogether four molecular descriptors were computed using Tango [35-37] and other 268 with JPEDES tool [17]. Those descriptors were extracted for each peptide sequence in the training and test datasets.

\section{Preprocessing}

We conducted a two-level preprocessing for the descriptor matrix previously generated. First, we applied a preprocessing at the instance level that consisted of removing outliers; these are vectors labeled with the same class that are very different from the rest, and that might affect the performance of chemical space characterization. Second, we applied a preprocessing at the descriptor level that renders all molecular descriptor values to the same range. This is because the employed molecular descriptors have different range values, e.g., the isoelectric point takes values in the order of $10^{0}$ to $10^{1} \mathrm{pH}$ units, whereas the molecular weight in the order of $10^{2}$ to $10^{3}$ Daltons.
To remove isolated vectors concerning their neighborhood, the Local Outlier Factor (LOF) [38] method was used. It should be noted that the LOF was applied to each class (e.g., AMP and non-AMP) from each dataset. Regarding preprocessing at the descriptor level, we applied the Min-Max scaling method, which maps the measures for each descriptor into a range of 1 to 10 [17]. As a result, we obtained a normalized descriptor matrix $\mathcal{D}$.

\section{Multi-objective evolutionary approach for feature weighting (MOEA-FW)}

The multi-objective evolutionary algorithm based on decomposition (MOEA/DDE) [29, 30] was employed to solve the multi-objective feature weighting problem earlier formulated (see Problem statement). The work in [30] shows that MOEA/D-DE performs better than the well known NSGAII [39] for continuous optimization problems, like the one described in this study (see Eq. 6).

In short, MOEA/D-DE decomposes the multi-objective optimization problem into $N$ single-objective optimization problems by adopting the Tchebycheff approach. Then, these $\mathrm{N}$ problems are solved simultaneously (for a detailed description of this method we refer to the interested reader to $[29,30])$.

In general, this algorithm receives as input the descriptor matrix $\mathcal{D}$ and gives as output a set of approximated $N$ optimal solutions to (6), this is called approximate Pareto set: $\mathcal{P}^{*}=\left\{\mathbf{w}^{\mathbf{1}}, \ldots, \mathbf{w}^{\mathbf{N}}\right\}$. It should be noted that each solution is a weight vector $\mathbf{w}^{\mathbf{k}}=\left[w_{1}^{k}, \ldots, w_{m}^{k}\right]^{T}$, where the $i$-th component is the scale factor for the $i$-th molecular descriptor. For each solution $\mathbf{w}^{\mathbf{k}}$ in $\mathcal{P}^{*}$, an objective vector $F\left(\mathbf{w}^{\mathbf{k}}\right)=\left[f_{1}\left(\mathbf{w}^{\mathbf{k}}\right), f_{2}\left(\mathbf{w}^{\mathbf{k}}\right)\right]^{T}$ is assigned. Then the set of all these objective vectors is called the approximate Pareto front [40]: $P F=\left\{F\left(\mathbf{w}^{\mathbf{1}}\right), \ldots, F\left(\mathbf{w}^{\mathbf{N}}\right)\right\}$.

It is important to note that, solutions in $\mathcal{P}^{*}$ cannot be considered better among themselves in both objectives since they are in a trade-off relation. This means that, some solutions in $\mathcal{P}^{*}$ are better in objective $f_{1}$ than in $f_{2}$ and vice-versa (see Fig. 5). To draw a few solutions from $\mathcal{P}^{*}$, taking into account different satisfiability levels of the objectives, we employed a well-established process in multi-criteria decision making [40].

\section{Multi-criteria decision making approach to select weight vectors}

For the problem of choosing a few weight vectors from the approximate Pareto set $\mathcal{P}^{*}$, we followed a process that receive as input $\mathcal{P}^{*}$. The main steps can be described as follows:

Step 1: for each solution, $\mathbf{w} \in \mathcal{P}^{*}$, scale the values for objective functions $f_{1}(\mathbf{w})$ and $f_{2}(\mathbf{w})$ to a range

between 0 and 1 , where 1 means full satisfaction for a 


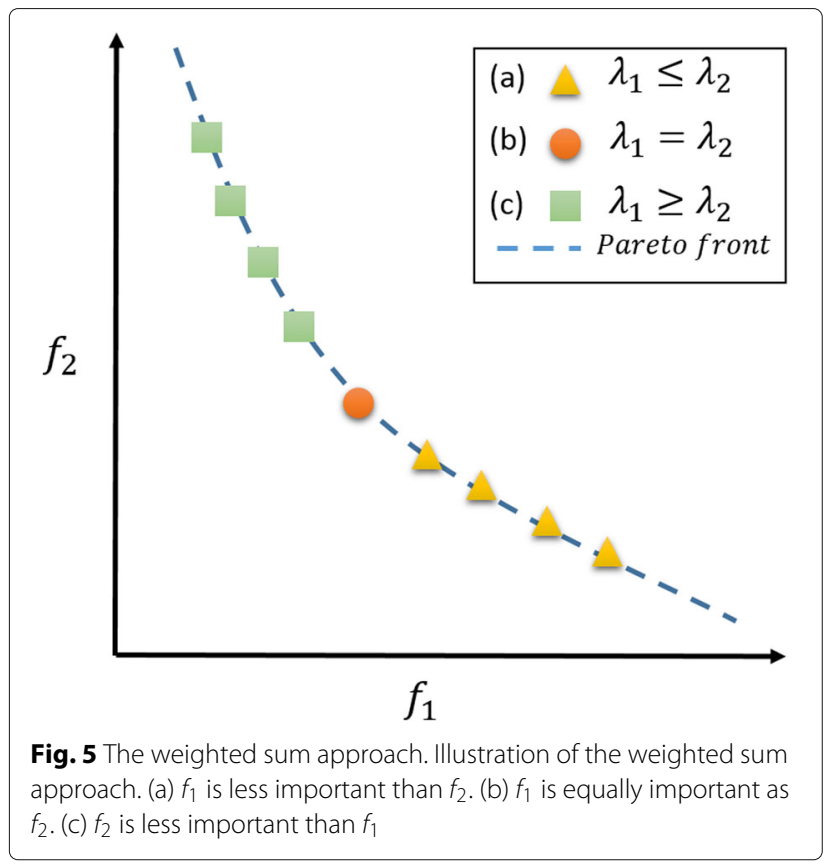

particular objective, and 0 indicates dissatisfaction. Perform the scaling of a solution $\mathbf{w}^{\mathbf{k}}$ for the objective $f_{i}$ as follows [16]:

$$
\mu_{i}^{k}= \begin{cases}1 & \text { if } f_{i}\left(\mathbf{w}^{\mathbf{k}}\right)=f_{i}^{\min }, \\ \frac{f_{i}^{\max }-f_{i}\left(\mathbf{w}^{\mathbf{k}}\right)}{f_{i}^{\max }-f_{i}^{\min }} & \text { if } f_{i}^{\min }<f_{i}^{k}<f_{i}^{\max }, \\ 0 & \text { if } f_{i}\left(\mathbf{w}^{\mathbf{k}}\right)=f_{i}^{\max },\end{cases}
$$

where,

$$
\begin{aligned}
& f_{i}^{\min }=\min _{1 \leq j \leq N}\left\{f_{i}\left(\mathbf{w}^{\mathbf{j}}\right)\right\}, \\
& f_{i}^{\max }=\max _{1 \leq j \leq N}\left\{f_{i}\left(\mathbf{w}^{\mathbf{j}}\right)\right\} .
\end{aligned}
$$

Here $\mu^{\mathbf{k}}=\left[\mu_{1}^{k}, \mu_{2}^{k}\right]^{T}$ is the objective vector constrained to the $[0,1]$ range for the solution $\mathbf{w}^{\mathbf{k}}$ in the approximate Pareto set $\mathcal{P}^{*}$.

Step 2: perform a weighted sum approach given a weight vector $\lambda=\left[\lambda_{1}, \lambda_{2}\right]^{T}$. Here $\lambda_{1}$ and $\lambda_{2}$ are used to set the preference over objectives $f_{1}$ and $f_{2}$, respectively. For instance, if we want a solution that satisfies $f_{1}$ more than $f_{2}$, then a greater value should be assigned to $\lambda_{1}$ than to $\lambda_{2}$ (see Fig. 5). Given $\lambda$, the weighted sum for each objective vector $\mu^{\mathbf{k}}$ is calculated as follows:

$$
g^{b c s}\left(\mu \mid \lambda_{1}\right)=\lambda_{1} \mu_{1}+\left(1-\lambda_{1}\right) \mu_{2}
$$

Step 3: Find the best compromise solution given $\lambda$ namely, the weight vector $\mathbf{w}^{k *}$ with the maximum value of $g^{b c s}$ (formally described in Eq. 1).

$$
k^{*}=\underset{k \in[1, N]}{\arg \max } g^{b c s}\left(\mu^{\mathbf{k}} \mid \lambda_{1}\right)
$$

In this work, for each dataset, we selected five of the best compromise solutions by using $\lambda_{1}$ equals to $0.4,0.45,0.5$, 0.55 , and 0.60 .

\section{Classification algorithms}

This section describes an assessment method to validate the performance of the MOEA-FW method. In this method, we evaluated the classification task before (i.e., baseline), and after applying our MOEA-FW algorithm. For each classification task, we built four models: random forest $(\mathrm{RF}), \mathrm{k}$-nearest neighbor $(\mathrm{KNN})$, a linear support vector machine (SVM-L), and a multi-layer perceptron (MLP). A training dataset without weight factors was used before applying our MOEA-FW algorithm, and the weighted molecular descriptors are used after that. Later, we compared the classification performance between the models and measured indirectly the quality of our proposal. To accomplish this, each best compromise solution (i.e., the weight vector $\mathbf{w}^{\mathbf{k} *}$ ) was applied to dataset $\mathcal{D}$ resulting in a new dataset $\hat{\mathcal{D}}_{\mathbf{W}}$, where:

$$
\hat{\mathcal{D}}_{\mathbf{W}}=\left[\begin{array}{cccc|c}
w_{1} x_{11} & w_{2} x_{12} & \cdots & w_{m} x_{1 m} & y_{1} \\
w_{1} x_{21} & w_{2} x_{22} & \cdots & w_{m} x_{2 m} & y_{2} \\
\vdots & & \ddots & \vdots & \vdots \\
w_{1} x_{n 1} & w_{2} x_{n 2} & \cdots & w_{m} x_{n m} & y_{n}
\end{array}\right]
$$

In this way, after applying our proposal, the rejected molecular descriptors correspond to columns whose values are zero and those columns were deleted.

\section{Implementation details}

All experiments were performed under the following condition; OS: ubuntu 16.04 LTS; CPU: Intel i7 at $2.40 \mathrm{GHz}$; and RAM memory: $12 \mathrm{~GB}$.

The MOEA/D-DE algorithm was implemented in Java using the framework of Metaheuristics for solving multiobjective optimization problems MOEA Framework 2.1 (available from http://www.moeaframework.org). The main parameters for MOEA/D-DE were set according to the values recommended in [30] for 2-objective problems, the specific parameter settings are summarized in [17].

The classification algorithms were implemented in Python 3.6 using the Scikit-learn [41]. Scikit-learn is an efficient set of tools for the implementation of machine learning algorithms for data mining tasks. The machine learning algorithms' hyperparameters are summarized as a following: $\mathrm{KNN}(p=1$, weight $=$ distance) and $k=19,22,3$ for the antimicrobial, 
antibacterial, and bacteriocin datasets, respectively; SVM$\mathrm{L}$ (class_weight $=$ balanced) and the penalty parameter $\mathrm{C}=0.001,0.1$, and 0.001 for the antimicrobial, antibacterial, and bacteriocin datasets, respectively; RF ( criterion=gini, max_features=sqrt); finally for MLP we used the default hyperparameters.

\section{Additional files}

Additional file 1: Performance comparison of the best compromise solutions given $\lambda_{1}$ for four different machine learning algorithms. The values are related to the average of the balance accuracy. (PDF $341 \mathrm{~kb}$ )

Additional file 2: Predictions of antimicrobial activity for DAMPD_AMP. Evaluation of different models with DAMP_AMP after applying the best compromise solutions and four machine learning algorithms. This file shows the results obtained by each of the best compromise solutions given $\lambda_{1}$ in each fold of the 10 -fold cross-validation test. (CSV $33 \mathrm{~kb}$ )

Additional file 3: Predictions of antimicrobial activity for APD3_AMP. Evaluation of different models with APD3_AMP after applying the best compromise solutions and four machine learning algorithms. This file shows the results obtained by each of the best compromise solutions given $\lambda_{1}$ in each fold of the 10 -fold cross-validation test. (CSV $34 \mathrm{~kb}$ )

Additional file 4: Predictions of antibacterial activity for DAMPD_ANTIBACTERIAL. Evaluation of different models with DAMPD_ANTIBACTERIAL after applying the best compromise solutions and four machine learning algorithms. This file shows the results obtained by each of the best compromise solutions given $\lambda_{1}$ in each fold of the 10-fold cross-validation test. (CSV $32 \mathrm{~kb}$ )

Additional file 5: Predictions of antibacterial activity for APD3_ANTIBACTERIAL. Evaluation of different models with APD3_ANTIBACTERIAL after applying the best compromise solutions and four machine learning algorithms. This file shows the results obtained by each of the best compromise solutions given $\lambda_{1}$ in each fold of the 10 -fold cross-validation test. (CSV $34 \mathrm{~kb}$ )

Additional file 6: Predictions of bacterocins for DAMPD_BACTERIOCIN. Evaluation of different models with DAMPD_BACTERIOCIN after applying the best compromise solutions and four machine learning algorithms. This file shows the results obtained by each of the best compromise solutions given $\lambda_{1}$ in each fold of the 10 -fold cross-validation test. (CSV $22 \mathrm{~kb}$ )

Additional file 7: Predictions of bacterocins for APD3_BACTERIOCIN. Evaluation of different models with APD3_BACTERIOCIN after applying the best compromise solutions and four machine learning algorithms. This file shows the results obtained by each of the best compromise solutions given $\lambda_{1}$ in each fold of the 10 -fold cross-validation test. (CSV $31 \mathrm{~kb}$ )

\section{Abbreviations}

10-fold CV: 10-fold Cross-Validation; Acc: Accuracy; AMPs: Antimicrobial peptides; APD3: Antimicrobial Peptide Database; AUC: Area under a ROC curve; Bal Acc: Balance accuracy; DAMPD: Dragon Antimicrobial Peptide Database; JPEDES: Java Peptide Descriptor from Sequences; KNN: k-nearest neighbor; LOF: Local Outlier Factor; MCC: Matthews correlation coefficient; MLAs: machine learning algorithms; MLP: Multi-layer perceptron; MOEA-FW: Multi-Objective Approach for Feature Weighting; MOEA/D-DE: Multi-objective evolutionary algorithm based on decomposition; MOP: Multi-objective optimization problem; Prec: Precision; QSAR: Quantitative Structure-Activity Relationship; RF: Random forest; Sens: Sensitivity; SVM-L: Linear support vector machine; VS: Virtual Screening

\section{Acknowledgements}

The authors would like to thank the anonymous reviewers for their valuable comments and suggestions to improve the quality of this paper.

\section{Funding}

Publications costs were funded by Center for Scientific Research and Higher Education of Ensenada (CICESE).

\section{About this supplement}

This article has been published as part of BMC Genomics Volume 19 Supplement 7, 2018: Selected articles from the IEEE BIBM International Conference on Bioinformatics \& Biomedicine (BIBM) 2017: genomics. The full contents of the supplement are available online at

https://bmcgenomics.biomedcentral.com/articles/supplements/volume-19supplement-7.

\section{Authors' contributions}

$J A B$ and $C A B$ defined the problem and designed the methodology; $J A B$ performed the experiments and analyzed the data; JAB, LAM and CAB. wrote the paper. JAB and CAB edited the final version. All authors read and approved the final manuscript.

\section{Ethics approval and consent to participate}

Not applicable.

\section{Consent for publication}

Not applicable.

\section{Competing interests}

The authors declare that they have no competing interests.

\section{Publisher's Note}

Springer Nature remains neutral with regard to jurisdictional claims in published maps and institutional affiliations.

\section{Published: 24 September 2018}

\section{References}

1. Cherkasov A, Hilpert K, Jenssen H, Fjell CD, Waldbrook M, Mullaly SC, Volkmer R, Hancock RE. Use of artificial intelligence in the design of small peptide antibiotics effective against a broad spectrum of highly antibiotic-resistant superbugs. ACS Chem Biol. 2008;4(1):65-74.

2. Stahura FL, Bajorath J. Partitioning methods for the identification of active molecules. Curr Med Chem. 2003;10(8):707-15.

3. Fjell CD, Hiss JA, Hancock RE, Schneider G. Designing antimicrobial peptides: form follows function. Nat Rev Drug Discov. 2012;11(1):37-51.

4. Kleandrova W, Ruso JM, Speck-Planche A, Dias Soeiro Cordeiro MN. Enabling the discovery and virtual screening of potent and safe antimicrobial peptides. simultaneous prediction of antibacterial activity and cytotoxicity. ACS Comb Sci. 2016;18(8):490-8.

5. Raventos D, Taboureau O, Mygind P, Nielsen J, Sonksen C, Kristensen $\mathrm{H}-\mathrm{H}$. Improving on nature's defenses: optimization \& high throughput screening of antimicrobial peptides. Comb Chem High Throughput Screen. 2005;8(3):219-33.

6. Jenssen H. Descriptors for antimicrobial peptides. Expert Opin Drug Discov. 2011;6(2):171-84.

7. Liu H, Motoda H. Feature Extraction, Construction and Selection: A Data Mining Perspective vol. 453; 1998.

8. Veltri $\mathrm{D}$, Kamath $\mathrm{U}$, Shehu A. Improving recognition of antimicrobial peptides and target selectivity through machine learning and genetic programming. IEEE/ACM Trans Comput Biol Bioinforma. 2017;14(2): 300-313.

9. Torrent M, Andreu D, Nogués VM, Boix E. Connecting peptide physicochemical and antimicrobial properties by a rational prediction model. PloS one. 2011;6(2):16968.

10. Waghu FH, Gopi L, Barai RS, Ramteke P, Nizami B, Idicula-Thomas S. Camp: Collection of sequences and structures of antimicrobial peptides. Nucleic Acids Res. 2014;42(D1):1154-8.

11. Fernandes FC, Rigden DJ, Franco OL. Prediction of antimicrobial peptides based on the adaptive neuro-fuzzy inference system application. Pept Sci. 2012;98(4):280-287.

12. Gabere MN, Noble WS. Empirical comparison of web-based antimicrobia peptide prediction tools. Bioinformatics. 2017;33(13):1921-1929.

13. Kohavi R, John GH. Wrappers for feature subset selection. Artif Intell. 1997;97(1):273-324. 
14. Dash M, Liu H. Feature selection for classification. Intell Data Anal. 1997;1(3):131-56.

15. Huang J, Cai $Y, X u X$. A hybrid genetic algorithm for feature selection wrapper based on mutual information. Pattern Recogn Lett. 2007;28(13): 1825-44.

16. Paul S, Das S. Simultaneous feature selection and weighting-an evolutionary multi-objective optimization approach. Pattern Recogn Lett. 2015;65:51-59.

17. Beltrán JA, Aguilera-Mendoza L, Brizuela CA. Feature weighting for antimicrobial peptides classification: A multi-objective evolutionary approach. In: 2017 IEEE Int Conf Bioinforma Biomed (BIBM); 2017. p. 276-283. IEEE.

18. Cai C, Gong J, Liu X, Gao D, Li H. Molecular similarity: methods and performance. Chin J Chem. 2013;31(9):1123-32.

19. Hocke J, Martinetz T. Maximum distance minimization for feature weighting. Pattern Recogn Lett. 2015;52:48-52.

20. Amaldi $E$, Kann V. On the approximability of minimizing nonzero variables or unsatisfied relations in linear systems. Theor Comput Sci. 1998;209(1):237-60.

21. Roy K, Kar S, Das RN. QSAR/QSPR Modeling: Introduction. Cham: Springer; 2015, pp. 1-36

22. Baldi P, Brunak S, Chauvin Y, Andersen CA, Nielsen H. Assessing the accuracy of prediction algorithms for classification: an overview. Bioinformatics. 2000;16(5):412-24.

23. Friedman M. A comparison of alternative tests of significance for the problem of m rankings. Ann Math Stat. 1940;11(1):86-92.

24. Demšar J. Statistical comparisons of classifiers over multiple data sets. J Mach Learn Res. 2006;7:1-30.

25. Lata S, Sharma B, Raghava G. Analysis and prediction of antibacterial peptides. BMC Bioinforma. 2007;8(1):263.

26. Lata S, Mishra NK, Raghava GP. Antibp2: improved version of antibacterial peptide prediction. BMC Bioinforma. 2010;11(1):19.

27. van Heel AJ, de Jong A, Montalban-Lopez M, Kok J, Kuipers OP. Bagel3: automated identification of genes encoding bacteriocins and (non-) bactericidal posttranslationally modified peptides. Nucleic Acids Res. 2013;41(W1):448-53.

28. Hammami R, Zouhir A, Hamida JB, Fliss I. Bactibase: a new web-accessible database for bacteriocin characterization. Bmc Microbiol. 2007;7(1):89.

29. Zhang Q, Li H. Moea/d: A multiobjective evolutionary algorithm based on decomposition. IEEE Trans Evol Comput. 2007;11(6):712-31.

30. Li H, Zhang Q. Multiobjective optimization problems with complicated pareto sets, moea/d and nsga-ii. IEEE Trans Evol Comput. 2009;13(2): 284-302.

31. Seshadri Sundararajan V, Gabere MN, Pretorius A, Adam S, Christoffels A, Lehväslaiho M, Archer JA, Bajic VB. Dampd: a manually curated antimicrobial peptide database. Nucleic Acids Res. 2011;40(D1): 1108-12.

32. Wang G, Li X, Wang Z. Apd3: the antimicrobial peptide database as a tool for research and education. Nucleic Acids Res. 2016;44(D1): 1087-1093.

33. Wang G, Li X, Zasloff $M$, et al. A database view of naturally occurring antimicrobial peptides: nomenclature, classification and amino acid sequence analysis; 2010. p. 1-21.

34. Todeschini R, Consonni V. Handbook of Molecular Descriptors vol. 11. New York: John Wiley \& Sons; 2008.

35. Rousseau F, Schymkowitz J, Serrano L. Protein aggregation and amyloidosis: confusion of the kinds? Curr Opin Struct Biol. 2006;16(1): 118-26.

36. Fernandez-Escamilla A-M, Rousseau F, Schymkowitz J, Serrano L. Prediction of sequence-dependent and mutational effects on the aggregation of peptides and proteins. Nat Biotechnol. 2004;22(10): 1302-6.

37. Linding R, Schymkowitz J, Rousseau F, Diella F, Serrano L. A comparative study of the relationship between protein structure and $\beta$-aggregation in globular and intrinsically disordered proteins. J Mol Biol. 2004;342(1): 345-53.

38. Breunig MM, Kriegel H-P, Ng RT, Sander J. Lof: identifying density-based local outliers. In: ACM Sigmod Record; 2000. p. 93-104. ACM.

39. Deb K, Pratap A, Agarwal S, Meyarivan T. A fast and elitist multiobjective genetic algorithm: Nsga-ii. IEEE Trans Evol Comput. 2002;6(2): 182-197.
40. Coello CAC, Lamont GB, Van Veldhuizen DA, et al. Evolutionary Algorithms for Solving Multi-objective Problems vol. 5. New York: Springer; 2007.

41. Pedregosa F, Varoquaux G, Gramfort A, Michel V, Thirion B, Grisel O, Blondel M, Prettenhofer P, Weiss R, Dubourg V, et al. Scikit-learn: Machine learning in python. J Mach Learn Res. 2011;12:2825-30.
Ready to submit your research? Choose BMC and benefit from:

- fast, convenient online submission

- thorough peer review by experienced researchers in your field

- rapid publication on acceptance

- support for research data, including large and complex data types

- gold Open Access which fosters wider collaboration and increased citations

- maximum visibility for your research: over $100 \mathrm{M}$ website views per year

At BMC, research is always in progress.

Learn more biomedcentral.com/submissions 